\title{
Developing a rule-driven clinical decision support system with an extensive and adaptative architecture
}

\author{
Liang Xiao \\ School of Computer Science \\ Hubei University of Technology \\ Wuhan, China \\ 1x@mail.hbut.edu.cn
}

\author{
Gráinne Cousins, Tom Fahey, \\ Borislav D Dimitrov \\ Department of General Practice \\ Royal College of Surgeons in Ireland \\ Dublin, Republic of Ireland
}

\author{
Lucy Hederman \\ Department of Computer Science \\ Trinity College Dublin \\ Dublin, Republic of Ireland
}

\begin{abstract}
Clinical guidelines are central to the implementation of clinical decision support systems (CDSSs). Addition or revision of clinical guidelines usually causes the (re-) development of new or existing CDSSs. The separate maintenance of clinical knowledge and their driving systems implies extra system development cost and low knowledge delivery efficiency. We propose, in this paper, an approach to liaise the two activities and support a complete knowledge-driven CDSS architecture. It will accommodate and disseminate new knowledge with minimum efforts required to make relevant changes to the systems, but make use of the new knowledge whenever it becomes available. A Multi-Agent System architecture and a rule-based knowledge repository are put together to realize this goal.
\end{abstract}

Keywords-clinical decision support system (CDSS); clinical guideline; evidence-based medicine; Model-Driven Architecture; Multi-Agent System; rule-based knowledge modelling

\section{INTRODUCTION}

Evidence-based medicine promotes conscious and explicit use of best external evidence in making clinical decisions [1] [2]. It involves a process to acquire, appraise, apply, and assess the evidence. Evidence may be gained from scientific studies and all the best evidence need to be evaluated in systematic reviews prior to consideration in clinical practice. Applying the best evidence to patients demands the development of clinical decision support systems (CDSSs) that matches evidence-based guidelines with patient conditions and generates customized recommendation. Clinical guidelines summarize the highest quality evidence about diagnosis, prognosis, therapy, and their cost-effectiveness. They usually describe, in connection with patient symptoms and signs, the decision points, judgment about findings, possible treatment options and their outcomes, being connected in flowcharts or described in text-based documents. If such guidelines are formally structured and standardized, they will help to raise the quality of care when followed properly.

Computerised clinical guidelines are central to the development of CDSS. Methods that support the representation of guidelines for machine execution include Arden Syntax [3][4], Guideline Interchange Format (GLIF) [5], PROforma [6] and so on. They all propose standard representation for capturing and formalizing guidelines to facilitate knowledge sharing. Since the knowledge of what's the best available evidence always improves as clinical research progresses, the best evidence today may never be the case tomorrow. To this end, there would usually be a need to extend or adapt previous CDSS to reflect the current new knowledge, or develop new CDSS to accommodate acquired knowledge in other clinical disciplines. The maintenance of clinical knowledge and that of systems driven by the knowledge in separate processes implies extra cost of development and poor knowledge delivery efficiency. There is lack of research in the design of an extensive and adaptive CDSS architecture that is capable of new knowledge accommodation and dissemination and let change reflects in system implementation whenever new knowledge becomes available. This knowledge to system mapping process shall be made as automatic as possible, in reducing the development cost and maintenance burden of the CDSS. Structuring knowledge in a pre-defined rule format may help to build such an extensive and adaptive CDSS architecture. In this paper, we assume web-based CDSS architecture starts from patient symptom and sign collection and finishes with diagnosis or treatment suggestions. Clinicians are guided through a set of web-based navigation pages to ask questions or get recommendation. A lightweight approach is proposed that drive context-sensitive tasks such as generation of question lists or suggestion of therapies and also control the entire decision support process at runtime via a rule-based guideline knowledge repository.

\section{APPROACH OVERVIEW}

We aim at setting up a structured rule-based knowledge repository that represents clinical guidelines. The rule repository shall be scalable to support extensive knowledge gathering and maintenance, across multiple clinical disciplines. It shall also be flexible in providing decision support to as many clinical areas as possible, wherever their corresponding rule sets become available. The core rule repository captures the essence of clinical knowledge, and is the most valuable asset to us. In order to support cost-effective evidence delivery, the transformation of such rules, from clinical guidelines and to decision aids, would be made as automatic as possible. This requires a means to turn human configurable clinical knowledge to computer process-able rule repository and then to decision support systems, realizing the evidence-based practice. 
The idea of rule-based guideline representation towards computerized evidence and machine processing reflects our previous work experience in a more general Software Engineering research method, the Agent-Oriented Model Driven Architecture [7][8]. It has three parts: 1) a set of business rules are employed to capture business requirements, 2) software agents are the main components of the target system and their behaviour is driven by the rule model dynamically at runtime to fulfill the desired requirements, 3) business experts are responsible for the maintenance of business rules to reflect new business needs and this will be reflected in new agent behavioral pattern immediately. Rules as such will have more comprehensive formalism than the simple if-then rules as used by Arden Syntax but details will be left for future discussion. The advantages of using such an approach are easy requirements implementation and maintenance, especially when the addition or change to such requirements is frequent. In some sense, clinical guidelines could be regarded as the actual requirements to implement (once for all) and maintain (ongoing process) in clinical decision support systems. If we would like a CDSS to be useful and able to encompass as many disease disciplines as possible, the addition of clinical guidelines will be the norm and existing ones always subject to expert revision, just as regular requirements are subject to change when business users raise new needs in a dynamic runtime environment. Therefore, the adaptation and application of Agent-Oriented Model Driven Architecture to the area of Health Informatics seems to be appropriate, and could bring benefits such as efficient knowledge management and costeffective system delivery.

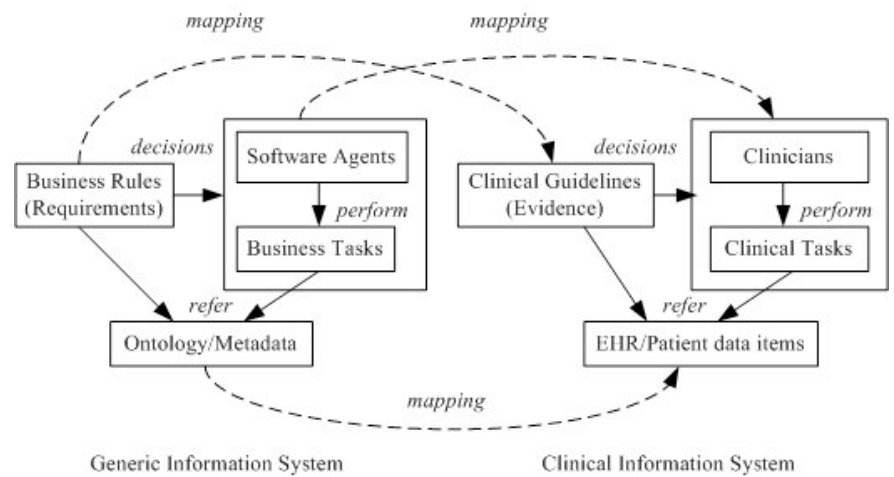

Figure 1. The mapping from a rule-driven software system to a guideline-driven clinical decision support system

Figure 1 shows the mapping schema or specializing from a rule-driven software system to an evidence-based clinical decision support system. Two important sorts of match support the proposal of such a schema. First, in a given information system, software agents must have the capabilities to realize requirements assigned to them, so let encapsulated business rules to drive their behaviour would help system management and maintenance. This matches the clinical scenario where clinical guidelines must be explicitly modeled and should be considered by clinicians when each individual uses their expertise to deliver care and make decisions. Second, business rules are composed by domain ontology or metadata, which helps semantic reasoning and automation of business task performance, for software agents. This matches the clinical scenario where clinical guidelines are matched with electronic patient health records (EHR) and customized recommendation is generated automatically, just at the point of care for clinicians. The above two kinds of match imply that methods and tools in the design of a rule-driven software architecture could be adapted for use in the clinical settings and similar advantages may be obtained. The fact that agents interpreting rules at runtime to reflect changing requirements is of most importance, as it liaises the maintenance of knowledgebase and the system driven by the knowledgebase in a single activity.

In the next section, we use a previously developed clinical decision support system for UTI as a vehicle to demonstrate what components a simple web-based CDSS may be made of, and how these may evolve into an extensive and adaptive knowledge-driven architecture.

\section{CASE STUDY}

Urine contains various fluids including some waste products but usually no bacteria. When bacterial infection occurs, either in the bladder or kidney and multiply in the urine, they may cause a urinary tract infection (UTI). A bladder infection or lower UTI (acute cystitis) is more common and less serious than a kidney infection or upper UTI (pyelonephritis). UTI can often be treated with antibiotics easily if there is no complication [9].

Common symptoms of a lower UTI are burning with urination (dysuria), frequency/urgency of urination, and no vaginal discharge. An upper UTI may also present with flank pain and fever [10][11]. Risk factors for complicated UTI include [12]: severe illness, over 65 years of age, abnormalities of renal tract anatomy and function, foreign body within the renal tract, immunocompromised patients, diabetes, pregnancy, persistent infection despite treatment. renal impairment.

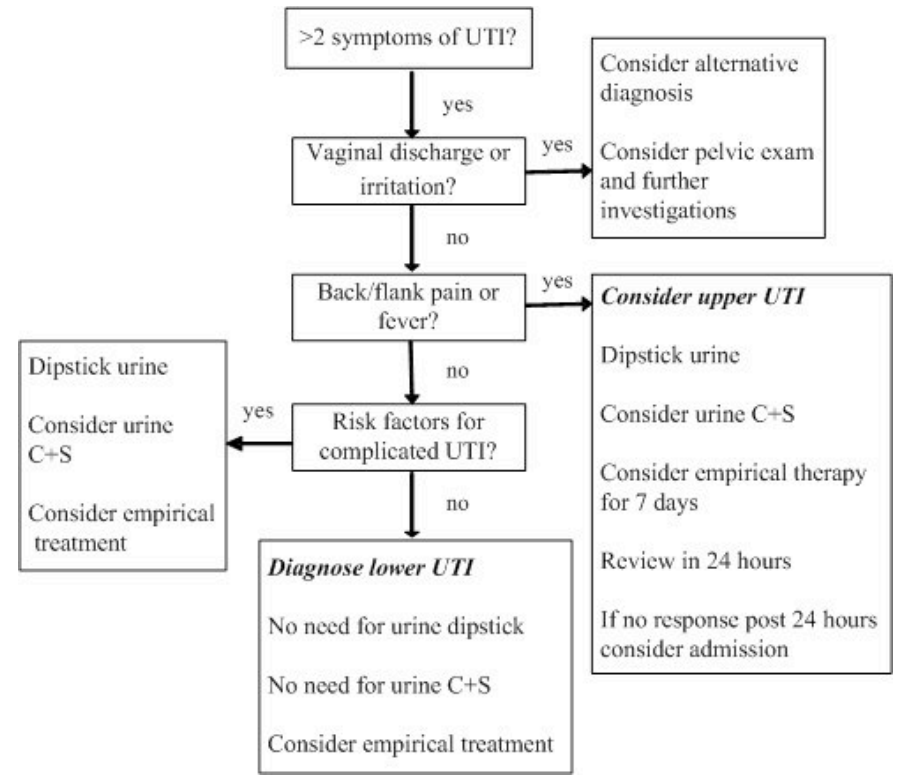

Figure 2. Clinical guidelines for UTI 
A diagnosis may be made and treatment given in many cases just based on symptoms without further laboratory investigation. In complicated situations, urine dipstick looking for the presence of urinary nitrites, leucocytes and the like as well as urine culture may be used to help diagnosis.

Clinical guidelines establishing the diagnosis of UTI for non pregnant woman can be summarized and shown above in Figure 2. A web-based CDSS that implements the guidelines consist of several steps, shown below from Figure 3 to Figure 6 . The mechanism of calculating diagnosis probability based on Bayesian network and likelihood ratio, as well as the generation of treatment options are omitted here for a concise description. First, the decision support system starts from the collection of present symptoms.

\begin{tabular}{|c|c|c|}
\hline Choose if appropriate & Symptom description & ICPC2 \\
\hline $\begin{array}{l}\text { () yes } \\
\text { O no }\end{array}$ & Dysuria & U01 \\
\hline $\begin{array}{l}\odot \text { ๖ yes } \\
\bigcirc \text { no }\end{array}$ & Urinary frequency & U02 \\
\hline $\begin{array}{l}\odot \text { yes } \\
\bigcirc \text { no }\end{array}$ & Haematuria & U06 \\
\hline $\begin{array}{l}\text { O yes } \\
\odot \text { no }\end{array}$ & Vaginal discharge & $\mathrm{X} 14$ \\
\hline $\begin{array}{l}\bigcirc \text { yes } \\
\odot \text { no }\end{array}$ & Vaginal irritation & \\
\hline $\begin{array}{l}\text { ○ yes } \\
\odot \text { no }\end{array}$ & Costovertebral angle tenderness & \\
\hline
\end{tabular}

Figure 3. The CDSS starts from asking about present symptoms

The probability of UTI diagnosis is calculated based on the presence of key symptoms. Next, further information needs to be collected for giving full management plans.

\footnotetext{
From the information we gathered so far, the probability of UTI diagnosis is: $96.44 \%$, we need a bit more information to give you full decision support 1. Back pain? $\bigcirc$ Yes $\odot$ No

2. Fever? $\odot$ Yes $\bigcirc$ No

3. Risk factors for complicated UTI? $\bigcirc$ Yes $\odot$ No

Risk factors for complicated UTI

- Serere illness, including hypotension, tachycardia, reduced levels of consciousness, or dehydration

- People older than 65 years of age.

- Abnormalities of renal tract anatomy and function (such as vesico-ureteric reflux, polycystic kidhey disease).

- Foreign body within the renal tract, including renal stones and urinary, ureteric, or nephrostomy catheters

- Immunocom.

- Pregnancy.

- Renal imparment

Next
}

Figure 4. Initial diagnosis result is give and additional questions are asked

Later on, a final probability is given and management plans suggested. Clinicians can accept the diagnosis (given a high probability of upper UTI in the example below) and proceed to prescribing for it. Presence of different symptom combination may give different diagnosis result, e.g. upper and lower UTI or the need of further investigation.
From all the information we gathered, the final probability of UTI diagnosis is: $97.2 \%$.

High probability of UTI (> $80 \%)$ : Consider empirical treatment without urine dipstick or urine culture.

Futher Advice: Consider upper UTI

- Consider empirical therapy for 7 days

- Review in 24 hours

- If no response post 24 hours consider admission

Accept this diagnosis and continue to prescribe for upper UTI

Figure 5.Final diagnosis result is given as well as management plans

Finally, prescribing choices and recommendation are presented, with relevant guideline details linkable in the interface for viewing and checking. Two options are available for prescribing: 1. accept one of the recommended medicines (with dosage and duration) according to clinical guidelines; or 2. choose an alternative medicine.

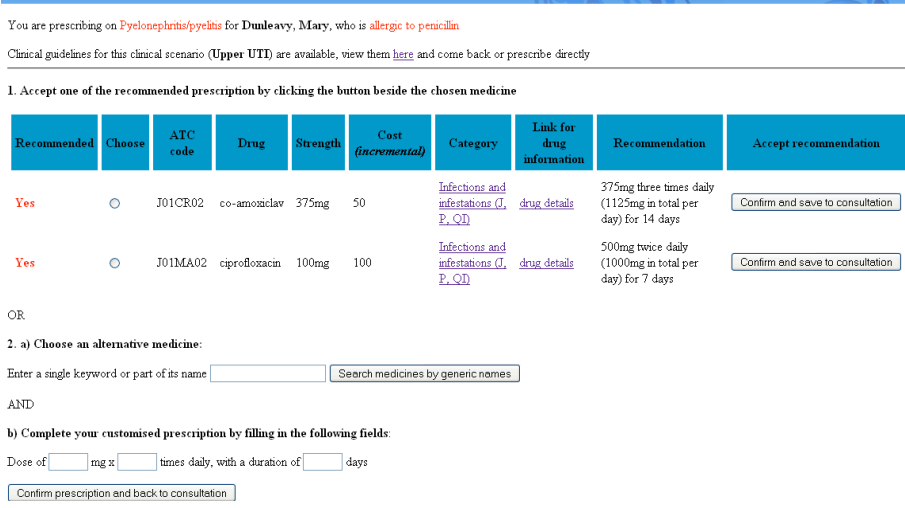

Figure 6. Recommendation of prescribing are offered

The user may simply accept a suggested drug and confirm the choice, this consultation with patient symptoms, diagnosis, and medication will be saved in the EHR. Alternative, the user may search the medicine database and choose one from them, give the dose and duration, and confirm this prescribing. Since this decision does not actually conform to the guidelines, confirmation is necessary, and both the current prescribing and the recommended one will be recorded for later analysis.

\section{TOWARDS AN ADAPTIVE AND EXTENSIVE RULE-DRIVEN DECISION SUPPORT SYSTEM ARCHITECTURE}

Imagining different clinical guidelines must be elicited and implemented in various disease areas, one may produce different clinical decision support systems accordingly. ICT and medical experts must work together continuously to transform clinical experience and evidence into practice, area by area. This is costly and inefficient.

Suppose a clinical guideline can be represented in a flowchart with condition check, decisions, and actions, as shown in Figure 2. Also let a clinical decision support system composed by a set of web-based pages, including symptom query, information collection, further investigation, and decision support provision, as shown from Figure 3 to Figure 6. We propose an approach of developing an extensive and 
adaptive clinical decision support system (CDSS) architecture that supports automation in two respects:

\section{A. Knowledge driven CDSS construction and presentation}

The construction and presentation of the clinical decision support systems will be entirely driven by the knowledgebase automatically on the fly, including information collection pages and recommendation generation pages. This is possible due to the fact that the determination of what questions shall be asked about the present symptoms or what conclusions shall be made about the diagnosis results depend upon the backend rule-based knowledge repository. Relevant information as such, structured via a pre-defined schema, may be dynamically populated into the frontend interfaces for presentation, guiding clinicians step by step. In addition, the availability of present patient conditions and guidelines together decide what further investigation will be necessary or what diagnosis results will be given, reflecting the state transition of the decision support process. This will also be determined at runtime, redirecting clinicians between different stages of the process, and enacting the generation and presentation of next pages. Thus, the rulebased knowledge repository will interact with clinicians via automatic generation of interfaces and control of processes via collecting what shall be known to it or giving what it can predict, all at runtime. This means there will be no need to redesign new systems for each new disease area when corresponding guidelines become available, but the gathering and structuring knowledge becomes the major goal.

\section{B. Knowledge structuring and formalising}

The rule-based knowledge repository across various disease disciplines generates questions and makes decisions in a unified manner. This means pre-defined syntax and semantics shall be followed so that the automatic CDSS construction and presentation for solving various problems becomes possible. Again, tools will be developed to support clinical experts to define clinical guidelines similar to what is shown in Figure 2, via flowchart drawing, condition checking, decision making, etc. as graphical representation. Such knowledge configuration tools later transform human readable knowledge representation into machine executable knowledge repository, in a rule-based structure and extendable across multiple disciplines. This means there will be no need to re-map new clinical guidelines to machine executable formats individually or manually.

The consequence of deploying the envisioned architecture is the support of addition or review of clinical guidelines in a clinician-friendly manner, as well as their availability to clinical users as clinical decision support systems immediately. The dissemination and reuse of clinical knowledge is made much easier. This demands work on the structuring of guidelines as semantic rich and strict rules as well as a mechanism of their interpretation, both in terms of graphical guidelines input and decision support interfaces output.

In developing the rule-based clinical decision support system architecture as such to promote automatic interface generation and regular clinical guidance, a Multi-Agent System (MAS) may be useful. As described in the second section of the paper, a comparison is made between a generic information system where rules decide how agents perform business tasks and a specific health information system where guidelines decide how clinicians perform clinical tasks. As different types of tasks emerge from the clinical decision support process, such as symptom collection, process control, and diagnosis/therapy generation, one may delegate different types of agents to accomplish corresponding tasks. A preliminary schema, which uses various agents each playing a certain kind of role, is given as follow.

- Query Agent.

Role: patient symptom query and information collection

- State Agent.

Role: state transition and process control

\section{- Knowledge Agent.}

Role: guideline knowledge management and diagnosis or therapy generation

The overall MAS architecture in implementing the envisioned rule-driven clinical decision support system with an automatic interface generation mechanism is shown below in Figure 7. Query Agent, State Agent, and Knowledge Agent together make up the MAS infrastructure supporting the realization of clinical decision support process. The actual CDSS as used by clinical users consists of Information Collection Pages, Page (re-) Direction, and Diagnosis/Therapy Decision Pages, which are categorized according to our experience accumulated via developing the UTI CDSS. The correlation between components of MAS and those of CDSS is as such: Query Agent retrieves questions to ask about patient symptoms from the Knowledge Agent and generates Information Collection Pages; State Agent keeps the current state of the decision support process and controls Page (re-) Direction; Knowledge Agent has access to the rule-based clinical guideline knowledge repository and is capable of making recommendation based on the collected symptoms and so it manages and generates Diagnosis/Therapy Decision Pages. Clinical experts input their knowledge and experience in form of flowchart-based guidelines to the knowledge repository via configuration tools. These tools will transform graphic guidelines to rule-based structures. The Knowledge Agent in the MAS will then be able to interpret knowledge from the rule repository in such a manner, and so helps automatic CDSS generation and implementation for guiding clinical users to make better decisions. Thus, this architecture supports knowledge dissemination or reuse via first transforming from human readable or editable knowledge model of clinical experts to the machine executable model of rule-based knowledge repository. Then the MAS executes the knowledge repository to support automatic CDSS process enactment and execution on the fly, transforming from the machine executable model to human executable model for use by regular clinical users. Consequently, the use of MAS supports rule-driven CDSS with an automatic knowledge-to-system generation architecture, offering an approach to implementing the evidenced-based medicine more efficiently. Gathering and structuring knowledge becomes the major tasks, clinical experts can focus on clinical problems, and IT developers are relieved from frequent development and maintenance burden. 


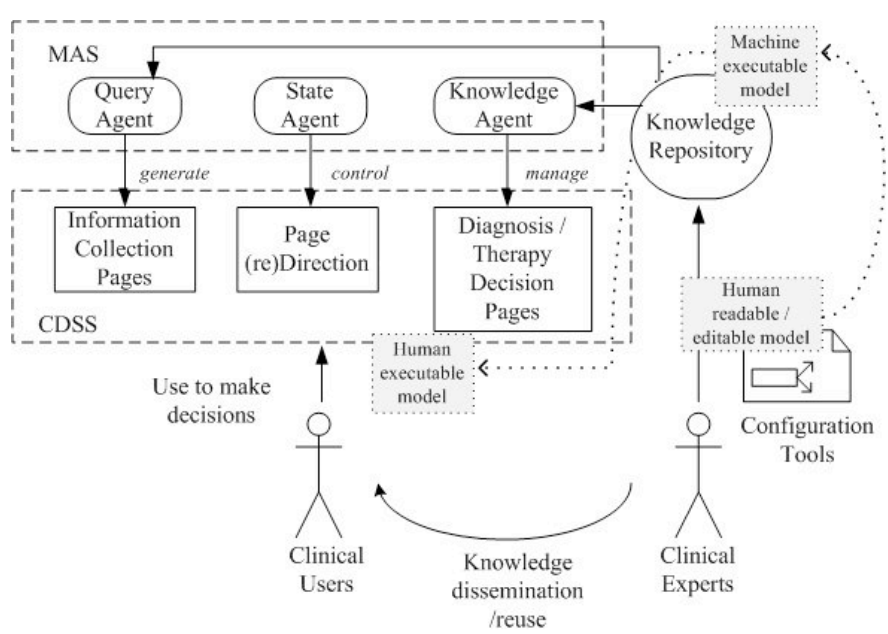

Figure 7. The use of MAS supports rule-driven CDSS with an automatic knowledge-to-system generation architecture

\section{DISCUSSION \& CONCLUSION}

We are confronted with today a large amount of data and knowledge that should be considered in clinical practice but often difficult to implement quickly and efficiently in clinical decision support tools, as being promoted by evidence-based medicine. A solution is described that proposes the use of a knowledge (evidences) repository, which supports mapping both from graphical knowledge configuration platform and to a knowledge driven decision support paradigm. The aim is to support better evidence maintenance and automatic system generation, when we consider clinical evidence across multiple clinical areas being in a similar executable formalism.

Two major components come into the picture to help the dissemination of clinical knowledge and evidences in such an automatic manner: 1) rule-based knowledge repository that takes in clinical guidelines and structures them in rules which predict the diagnosis or therapy based on symptoms according to widely-recognised evidence. Decision or recommendation will be given on a patient-specific basis; 2) multi-agent system enables knowledge transformation from machine executable rule structures to the decision support system enactment at runtime, as well as the determination of the final clinical conclusions that drawn upon from them.

Future work includes more detailed design of the MAS architecture, the rule structure, and the implementation of fully automatic CDSS generation mechanism. Also, tools will be developed to support the transformation from human readable graphical clinical guidelines to machine executable rule-based knowledge repository. The previously developed CDSS for UTI will be reconstructed following the proposed architecture as the first vehicle to demonstrate the feasibility and effectiveness of the approach. Finally an evaluation will be given to assess the value of the new architecture.

\section{ACKNOWLEDGMENT}

This work is supported by National Natural Science Foundation of China under Grant Number 61151001 and Health Research Board of Ireland through the HRB Centre for Primary Care Research under Grant HRC/2007/1.

\section{REFERENCES}

[1] Sackett, D.L., Rosenberg, W.M.C., Gray, J.A.M, Haynes, R.B., Richardson, W.S., "Evidence based medicine: what it is and what it isn't”, BMJ 312(71), 1996.

[2] Timmermans, S., Mauck, A., "The promises and pitfalls of evidencebased medicine". Health Aff (Millwood) 24 (1): 18-28, 2005.

[3] Hripcsak, G., Ludemann, P., Pruor, T.A., Wigertz, O.B., Clayton, P.B., "Rationale for the Arden Syntax", Computers in Biomedical Research 27 (4): 291-324, 1994

[4] Hripcsak, G., "Tutorial on how to use the Arden Syntax. Writing Arden Syntax medical logic modules", Computers in Biology and Medicine 24(5):331-63, 1994.

[5] Peleg, M., et al., "GLIF3: The Evolution of a Guideline Representation Format”, Proc AMIA Symp. 645-649, 2000.

[6] Sutton, D.R., Fox, J., "The Syntax and Semantics of the PROforma Guideline Modeling Language", Journal of American Medical Informatics Association 10(5):433-443, 2003.

[7] Xiao, L., Greer, D., “Adaptive Agent Model: Software Adaptivity using an Agent-oriented Model Driven Architecture", Information \& Software Technology 51(1): 109-137, Elsevier, 2009.

[8] Xiao, L., Greer, D., "Towards Agent-oriented Model-Driven Architecture", European Journal of Information Systems 16(4):390-406, Special Issue on Model-Driven Systems Development, Palgrave Macmillan, 2007.

[9] Zalmanovici, T.A., Green, H., Paul, M., Yaphe, J., Leibovici, L. "Antimicrobial agents for treating uncomplicated urinary tract infection in women" In Zalmanovici Trestioreanu, Anca "Cochrane Database of Systematic Reviews", Cochrane Database Syst Rev 10 (10): CD007182, 2010 .

[10] SIGN, "Management of suspected bacterial urinary tract infection in adults: a national clinical guideline", Scottish Intercollegiate Guidelines Network, www.sign.ac.uk, 2006.

[11] Nicolle L.E., "Uncomplicated urinary tract infection in adults including uncomplicated pyelonephritis". Urol Clin North Am 35 (1): 1-12, v., 2008.

[12] European Association of Urology, Guidelines on the management of urinary and male genital tract infections, European Association of Urology, www.uroweb.org. 2008. 